Hydrogen storage in engineered carbon nanospaces

This article has been downloaded from IOPscience. Please scroll down to see the full text article.

2009 Nanotechnology 20204026

(http://iopscience.iop.org/0957-4484/20/20/204026)

View the table of contents for this issue, or go to the journal homepage for more

Download details:

IP Address: 128.206.162.204

The article was downloaded on 04/06/2010 at 16:10

Please note that terms and conditions apply. 


\title{
Hydrogen storage in engineered carbon nanospaces
}

\author{
Jacob Burress ${ }^{1,2}$, Michael Kraus ${ }^{1,2}$, Matt Beckner ${ }^{1,2}$, \\ Raina Cepel $^{1,2}$, Galen Suppes ${ }^{1,3}$, Carlos Wexler ${ }^{1,2,4}$ and \\ Peter Pfeifer ${ }^{1,2}$ \\ ${ }^{1}$ Alliance for Collaborative Research in Alternative Fuel Technology, University of Missouri, \\ Columbia, MO 65211, USA \\ ${ }^{2}$ Department of Physics and Astronomy, University of Missouri, Columbia, MO 65211, USA \\ ${ }^{3}$ Department of Chemical Engineering, University of Missouri, Columbia, MO 65211, USA \\ E-mail:wexlerc@missouri.edu
}

Received 31 October 2008, in final form 30 January 2009

Published 24 April 2009

Online at stacks.iop.org/Nano/20/204026

\begin{abstract}
It is shown how appropriately engineered nanoporous carbons provide materials for reversible hydrogen storage, based on physisorption, with exceptional storage capacities $\left(\sim 80 \mathrm{~g} \mathrm{H}_{2} / \mathrm{kg}\right.$ carbon, $\sim 50 \mathrm{~g} \mathrm{H}_{2} /$ liter carbon, at 50 bar and $77 \mathrm{~K}$ ). Nanopores generate high storage capacities (a) by having high surface area to volume ratios, and (b) by hosting deep potential wells through overlapping substrate potentials from opposite pore walls, giving rise to a binding energy nearly twice the binding energy in wide pores. Experimental case studies are presented with surface areas as high as $3100 \mathrm{~m}^{2} \mathrm{~g}^{-1}$, in which $40 \%$ of all surface sites reside in pores of width $\sim 0.7 \mathrm{~nm}$ and binding energy $\sim 9 \mathrm{~kJ} \mathrm{~mol}^{-1}$, and $60 \%$ of sites in pores of width $>1.0 \mathrm{~nm}$ and binding energy $\sim 5 \mathrm{~kJ} \mathrm{~mol}^{-1}$. The findings, including the prevalence of just two distinct binding energies, are in excellent agreement with results from molecular dynamics simulations. It is also shown, from statistical mechanical models, that one can experimentally distinguish between the situation in which molecules do (mobile adsorption) and do not (localized adsorption) move parallel to the surface, how such lateral dynamics affects the hydrogen storage capacity, and how the two situations are controlled by the vibrational frequencies of adsorbed hydrogen molecules parallel and perpendicular to the surface: in the samples presented, adsorption is mobile at $293 \mathrm{~K}$, and localized at $77 \mathrm{~K}$. These findings make a strong case for it being possible to significantly increase hydrogen storage capacities in nanoporous carbons by suitable engineering of the nanopore space.
\end{abstract}

(Some figures in this article are in colour only in the electronic version)

\section{Introduction}

The development of a viable hydrogen-based economy depends on overcoming numerous scientific and technological barriers. In particular, for vehicular use, the US Department of Energy (DOE) has identified three major challenges to the successful adoption of hydrogen-powered vehicles $[1,2]$ : (i) hydrogen production costs must be substantially lowered (and long-term renewable sources for the energy required for its production must be identified and developed); (ii) a substantial reduction in fuel-cell costs (using hydrogen

4 Author to whom any correspondence should be addressed. in internal combustion engines significantly lowers their efficiency, compounding problems for production and storage); and (iii) hydrogen storage systems capable of delivering a driving range of hundreds of kilometers, without major detrimental effects to vehicle cost, safety or cargo capacity. Of the three challenges, perhaps the most difficult to solve is the development of on-board storage due to the various constraints imposed onto the system (cost and safety, volume and mass limitations, operation over a wide range of temperatures, charging times, reusability of the materials, etc). The problem for storage is that, at ambient conditions, unlike gasolineor alcohol-based liquid fuels, hydrogen is a gas with a very 
Table 1. Validation of $\mathrm{H}_{2}$ storage in corncob-based nanoporous carbon in independent laboratories. Temperatures departing from the ones in the table header are listed in parentheses.

\begin{tabular}{llll}
\hline & $77 \mathrm{~K}, 50 \mathrm{bar}$ & $293 \mathrm{~K}, 50 \mathrm{bar}$ & $293 \mathrm{~K}, 80 \mathrm{bar}$ \\
\hline Batch 5.1, U. Missouri & - & $1.0-1.1 \mathrm{wt} \%$ & - \\
& & $4.2-4.6 \mathrm{~g} \mathrm{l}^{-1}$ & \\
Batch 5.1, Hiden HTP1 (figure 1) & $7.0 \mathrm{wt} \%(83 \mathrm{~K})$ & $1.1 \mathrm{wt} \%(303 \mathrm{~K})$ & $1.8 \mathrm{wt} \%(303 \mathrm{~K})$ \\
& $29 \mathrm{~g} \mathrm{l}^{-1}$ & $4.6 \mathrm{~g} \mathrm{l}^{-1}$ & $7.6 \mathrm{~g} \mathrm{l}^{-1}$ \\
S-33/k, U. Missouri & $7.8-9.1 \mathrm{wt} \%$ & $0.9-1.1 \mathrm{wt} \%$ & - \\
& $45-53 \mathrm{~g} \mathrm{l}^{-1}$ & $5.2-5.7 \mathrm{~g} \mathrm{l}^{-1}$ & \\
S-33/k, Hiden IGA-001 (figure 1) & $7.6 \mathrm{wt}^{\mathrm{a}}$ & $1.1 \mathrm{wt}^{\mathrm{a}}$ & $1.6 \mathrm{wt}^{\mathrm{a}}{ }^{\mathrm{a}}$ \\
& $40 \mathrm{~g} \mathrm{l}^{-1}$ & $5.7 \mathrm{~g} \mathrm{l}^{-1}$ & $8.3 \mathrm{~g} \mathrm{l}^{-1}$ \\
\hline
\end{tabular}

${ }^{\text {a }}$ Values extrapolated from isotherms (equations (6) and (10)).

low energy density per unit volume. Traditionally, this was solved either by liquefaction at cryogenic conditions (e.g., as used in spacecraft), or by storing the gas at very high pressures (e.g., as used in some test vehicles). Besides the practical difficulties of storing hydrogen at such extreme conditions, a significant amount of energy is lost during liquefaction or compression $\left(\sim 1 / 3\right.$ and $\sim 1 / 5$ of the heating value of $\left.\mathrm{H}_{2}\right)$. Adsorbent-based storage materials, therefore, show promise because of their reversibility, ease of fueling, and absence of thermal management issues; however, no material presently comes close to the 2010 DOE targets of $60 \mathrm{~g} \mathrm{H}_{2} / \mathrm{kg}$ tank and $45 \mathrm{~g} \mathrm{H}_{2} /$ liter tank at low-pressure, room-temperature conditions.

Carbon-based materials, due to their low cost and weight, have long been considered as suitable physisorption substrates for the reversible storage of hydrogen, but the low $\mathrm{H}_{2}$ carbon interaction energies of ca. $5 \mathrm{~kJ} \mathrm{~mol}^{-1}$ result in disappointingly small storage capacity at room temperature for most carbon-based materials to date [3]. However, significantly higher storage can be achieved in activated carbon by surface functionalization: (i) via creation of nanometer sized pores that both dramatically raise the surface area for adsorption (3100 $\mathrm{m}^{2} \mathrm{~g}^{-1}$, or more), and create deeper potential wells (almost doubling the adsorption energy) [4]; and/or (ii) by doping with elements capable of strengthening the hydrogen-substrate interaction (boron, iron, nitrogen, intercalated fluoride ions, $\mathrm{Pt}, \mathrm{Pd}$, etc) [5].

This paper shows experimentally and computationally that the binding energy of $\mathrm{H}_{2}$-carbon systems can be significantly raised entirely on the basis of appropriate engineering of pore spaces at the nanometer scale. Section 2 presents the main experimental results for $\mathrm{H}_{2}$ adsorption at cryogenic and ambient temperatures for two activated carbon samples based on ground corncob (an agricultural waste product), showing storage capacities up to $100 \mathrm{~g} \mathrm{H}_{2} / \mathrm{kg}$ (90 bar, $83 \mathrm{~K}$ ) and $20 \mathrm{~g}$ $\mathrm{H}_{2} / \mathrm{kg}$ (90 bar, $303 \mathrm{~K}$ ). Section 3 describes the determination of absolute and excess adsorption and total amount stored. Section 4 shows, from first-principles statistical mechanical models (localized and mobile adsorption), that it is possible to discriminate experimentally between two vastly opposed situations of how easily molecules move along the surface. This will demonstrate that such lateral dynamics (competition of vibrational and translational degrees of freedom) affects the $\mathrm{H}_{2}$ storage capacity, and that control of such dynamics may offer new venues to increase storage capacities by as much as a factor of two. In section 5, it is shown that a good agreement between experimental and theoretical adsorption isotherms is possible based on a two-binding-energy model. A combination of statistical mechanical analysis and simulations demonstrates that the abundance of nanopores $(<1 \mathrm{~nm}$ in diameter) does not substantially reduce the rate at which a $\mathrm{H}_{2}$ tank can be filled or discharged (section 6). Finally (section 7), the requisite structural information on the structure of nanospaces, resembling exfoliated graphite, is obtained from $\mathrm{N}_{2}$ adsorption, non-local density functional theory, and small-angle $\mathrm{x}$-ray scattering.

\section{Hydrogen adsorption in nanoporous carbon: main experimental results}

Nanoporous carbon were fabricated by controlled pyrolysis of ground corncob using a proprietary multi-step method [6]. All samples under consideration in this paper were prepared in granular form. Hydrogen $(99.999 \%$ pure, run through a Matheson Trigas 450B gas purifier) uptakes were measured gravimetrically and volumetrically as excess adsorption, $m_{\text {ads }}^{\mathrm{e}}$ (total mass of $\mathrm{H}_{2}$ in the pore space minus the mass of bulk $\mathrm{H}_{2}$ that would be present in the absence of adsorption) on a custom-built system at the University of Missouri, Hiden IGA001 sorption analyzer (Hiden Isochema Ltd), and Hiden HTP1 sorption analyzer (Hiden Isochema Ltd). Excess adsorption was converted into total amount stored, $m_{\mathrm{st}}$ (mass of adsorbed and non-adsorbed hydrogen in the pore space), using

$$
m_{\mathrm{st}}=m_{\mathrm{ads}}^{\mathrm{e}}+\left(\rho_{\mathrm{a}}^{-1}-\rho_{\mathrm{s}}^{-1}\right) \rho_{\mathrm{gas}} m_{\mathrm{s}},
$$

where $\rho_{\mathrm{a}}, \rho_{\mathrm{s}}, \rho_{\text {gas }}$, and $m_{\mathrm{s}}$ are the apparent density of the sample (including pore space), skeletal density of the sample (without pore space), density of bulk gas, and mass of the sample, respectively. Figure 1 shows excess adsorption (direct experimental result) and total amount stored (calculated from equation (1), with $\rho_{\mathrm{a}}=0.58$ and $0.42 \mathrm{~g} \mathrm{~cm}^{-3}$ for $\mathrm{S}-33 / \mathrm{k}$ and Batch 5.1, respectively; and $\rho_{\mathrm{s}}=2.0 \mathrm{~g} \mathrm{~cm}^{-3}$ ). Detailed results, validated in independent laboratories, are presented in table 1, comparing favorably with the best carbons in the literature [7-9].

\section{Hydrogen adsorption: measures of adsorption}

Three different measures of adsorption are commonly found in the literature and often confused: absolute adsorption, excess 

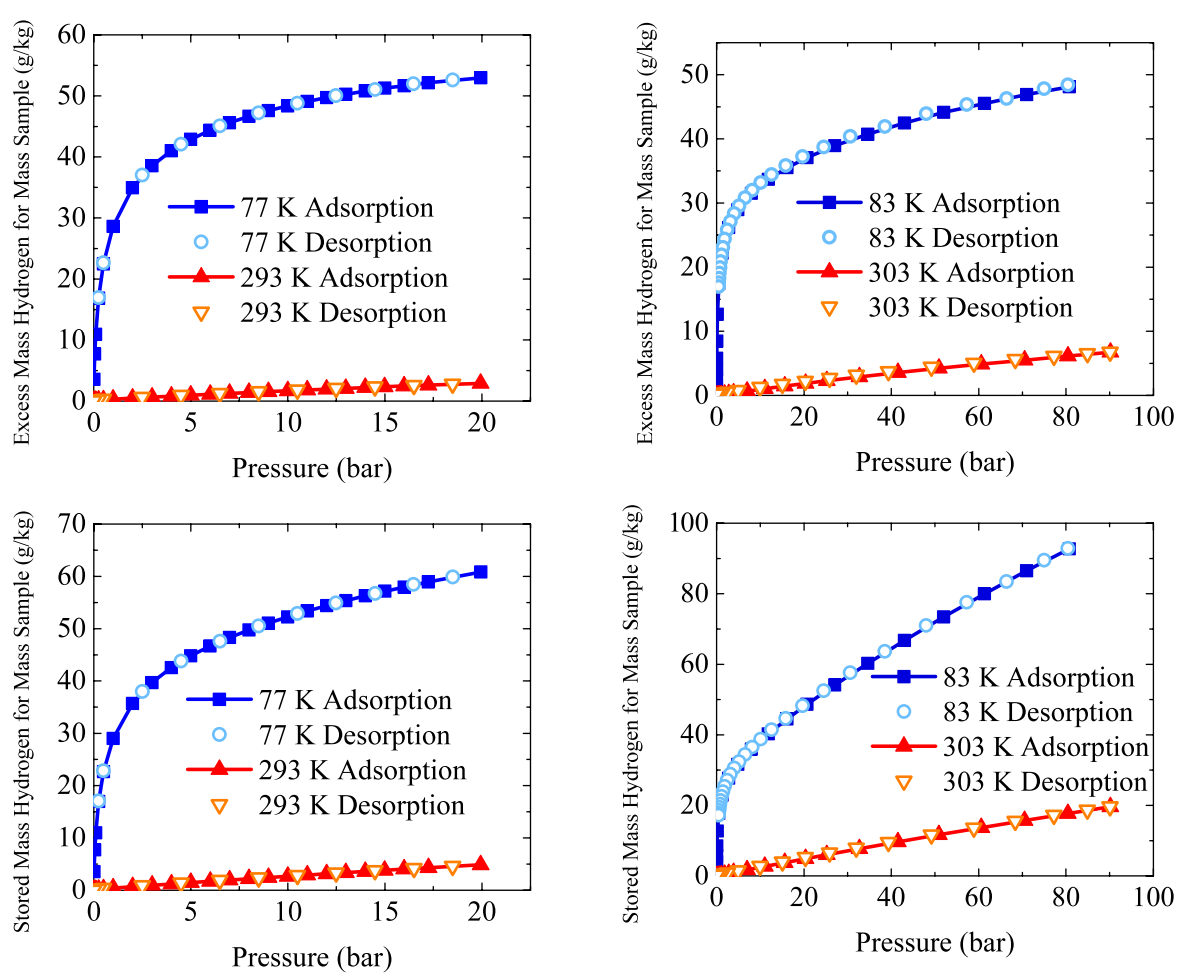

Figure 1. Excess hydrogen adsorption (top) and total amount stored (bottom) for samples S-33/k (left), and Batch 5.1 (right). The adsorption/desorption branches retrace each other, showing that the process is highly reversible.

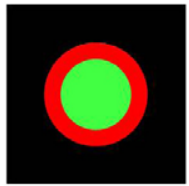

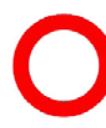

(b)

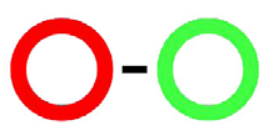

(c)

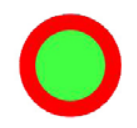

(d) (a)

Figure 2. (a) Cartoon depicting pore with adsorbed ring (dark red) and bulk gas inner circle (light green), (b) absolute adsorption, (c) excess adsorption and (d) amount stored.

adsorption and total amount stored. Absolute adsorption is defined to quantify the amount of adsorbate exclusively on the surface of the adsorbent. This quantity is often used because it is the direct output of all statistical mechanics theories and of all molecular simulations [10]. In contrast, excess adsorption is defined as the number of admolecules on the surface, minus the number of gas molecules that would have occupied this same volume in absence of adsorbentadsorbate attraction. Experimentally, only excess adsorption can be directly determined in either gravimetric or volumetric measurements [10]. Whereas at low pressures excess and absolute adsorption are similar, at high pressures they can differ significantly (in fact, excess adsorption at high pressures even decreases). Last, is the quantity of most interest from a practical point of view: the total amount stored, defined to include both adsorbed and non-adsorbed molecules in the porous materials. Figure 2 illustrates the differences between these different measures of adsorption.

For supercritical fluids, one can safely assume at most a monolayer of coverage, which yields a surface coverage of
$\theta=N / M$, where $N$ is the number of $\mathrm{H}_{2}$ molecules adsorbed on a surface with $M$ adsorption sites (absolute adsorption; no more than one molecule per surface site; $0 \leqslant \theta \leqslant 1$ ). To convert the number of adsorbed $\mathrm{H}_{2}$ molecules per number of adsorption sites, $\theta(p, T)$, into mass of adsorbed $\mathrm{H}_{2}$, $m_{\text {ads }}(p, T)$ ('absolute adsorption'), mass of excess adsorbed $\mathrm{H}_{2}$, ('excess adsorption'), and mass of stored $\mathrm{H}_{2}, m_{\mathrm{st}}(p, T)$ ('total amount stored'), per mass of sample, $m_{\mathrm{s}}$, requires some structural data of the sample. The data are: surface area of sample per mass of sample, $\Sigma$ (specific surface area); volume of nanopores, $V_{\text {nanopores }}$, hosting the adsorbed film; total pore volume, $V_{\text {all pores }}$; skeletal density of sample $\rho_{\mathrm{s}}$ (sample density with no pores included); and monolith density, $\rho_{\mathrm{a}}$ (sample density with all pores included). The adsorption measures of interest can be related by:

$$
\begin{aligned}
& \frac{m_{\mathrm{ads}}(p, T)}{m_{\mathrm{s}}}=\frac{\theta(p, T) m \Sigma}{\alpha(T)}, \\
& \frac{m_{\mathrm{ads}}^{\mathrm{e}}(p, T)}{m_{\mathrm{s}}}=\frac{m_{\mathrm{ads}}(p, T)}{m_{\mathrm{s}}}-\frac{V_{\text {nanopores }}}{m_{\mathrm{s}}} \rho_{\mathrm{H}_{2}, \mathrm{gas}}(p, T), \\
& \frac{m_{\mathrm{st}}(p, T)}{m_{\mathrm{s}}}=\frac{m_{\mathrm{ads}}(p, T)}{m_{\mathrm{s}}} \\
& \quad+\left(\frac{1}{\rho_{\mathrm{a}}}-\frac{1}{\rho_{\mathrm{s}}}-\frac{V_{\text {nanopores }}}{m_{\mathrm{s}}}\right) \rho_{\mathrm{H}_{2}, \text { gas }}(p, T), \\
& \rho_{\mathrm{H}_{2}, \text { gas }}(p, T)=\frac{p m}{N_{\mathrm{A}} k T} .
\end{aligned}
$$

The structural data used to implement (2)-(4), was obtained from nitrogen adsorption data at $77 \mathrm{~K}$ (Quantachrome Autosorb-1-C). The surface area and pore volumes were obtained from density functional analysis of the nitrogen 

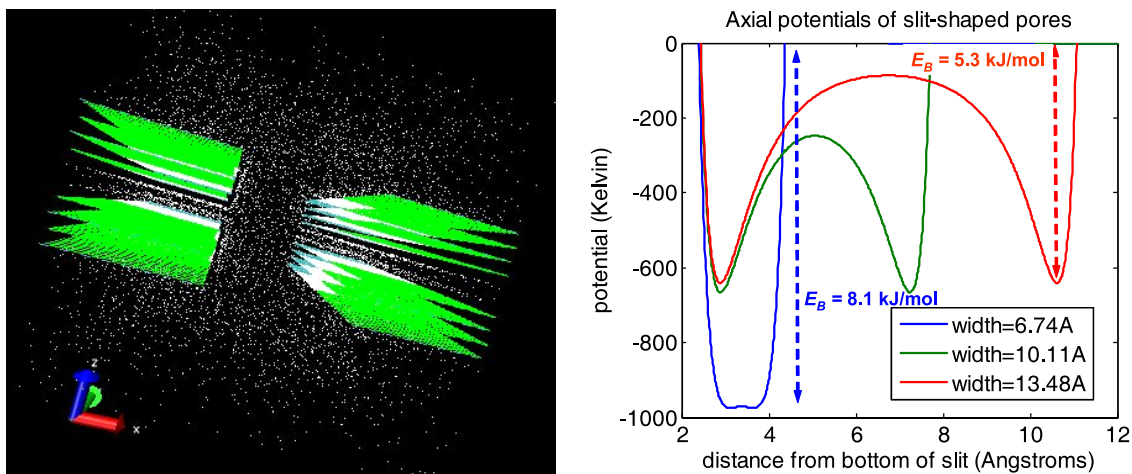

Figure 3. Left: simulation cell for a $\sim 10 \AA$ slit-shaped pore. Carbons in graphite are shown in green, $\mathrm{H}_{2}$ molecules in white. Note the high density of $\mathrm{H}_{2}$ near the surface. Right: laterally-averaged potential energy for $\mathrm{H}_{2}$ in slit-shaped pores. For narrow pores, the binding energy is enhanced due to the combined adsorption potentials from both sides of the pore.

adsorption/desorption isotherm (section 7), and $\rho_{\mathrm{a}}=$ $0.5 \mathrm{~g} \mathrm{~cm}^{-3}, \rho_{\mathrm{s}}=2.0 \mathrm{~g} \mathrm{~cm}^{-3}$.

\section{Computational and theoretical work: adsorption in slit-shaped pores and comparison between localized and mobile adsorption}

Theoretical predictions of $\mathrm{H}_{2}$ storage capacities under two distinct scenarios-localized and mobile adsorption-were carried out using the Langmuir adsorption isotherm and molecular dynamics (MD) simulations. Using the highly parallelized NAMD2 code [11], the simulations were carried out in the canonical ensemble $\mathrm{e}^{5}$ in a computational cell of size of approximately $100 \AA \times 100 \AA \times 100 \AA$. Graphene layers were introduced to simulate adsorption onto a graphite plate (6 layers) or onto slit-shaped pores (figure 3, left). Molecules interact via Lennard-Jones potentials with each other and with the carbon atoms in the graphite $[9,13]$. A total of $27 \leqslant N \leqslant$ $27581 \mathrm{H}_{2}$ molecules were used at each temperature, the time step for the integrations was $\Delta t=1 \mathrm{fs}$, and $\sim 10^{6}$ time steps were used for each simulation. The sum of the Lennard-Jones potentials between a $\mathrm{H}_{2}$ molecule and all carbon atoms gives an adsorption potential $V(x, y, z)$ with a strongly $z$-dependent attractive part (figure 3, right) and a weakly $(x, y)$-dependent corrugation part. Using the experimental binding energy of $5.0 \mathrm{~kJ} \mathrm{~mol}^{-1}$ for $\mathrm{H}_{2}$ on graphite [13], results in approximately $0.5 \mathrm{~kJ} \mathrm{~mol}^{-1}$ for the peak-to-peak amplitude of the corrugation potential. Characteristically, $\mathrm{H}_{2}$ binding energies are observed to range from $E_{\mathrm{B}} \cong 5.3 \mathrm{~kJ} \mathrm{~mol}^{-1}$ for the widest pores (similar to what is expected on the surface of graphite) to $E_{\mathrm{B}} \cong$ $8.1 \mathrm{~kJ} \mathrm{~mol}^{-1}$ for narrow pores, in good agreement with the two-binding-energy fits to experimental adsorption isotherms (section 5). Figure 4 depicts the laterally averaged density profile for two different pore widths. Simulated adsorption isotherms (figure 5) were computed for various pairs $(N, T)$, and equivalently for various pairs $(P, T)$ and compared to experimental absolute adsorption isotherms for sample $S-33 / \mathrm{k}$ (a surface area $\Sigma=2100 \mathrm{~m}^{2} \mathrm{~g}^{-1}$ was used to convert $\mathrm{H}_{2} / \AA^{2}$ to $\mathrm{g} / \mathrm{kg}$ ). The agreement is qualitative only, because $\mathrm{S}-33 / \mathrm{k}$ has

5 MD simulations included a 'thermostat' to keep temperature constant, see, e.g. [12], and [11].

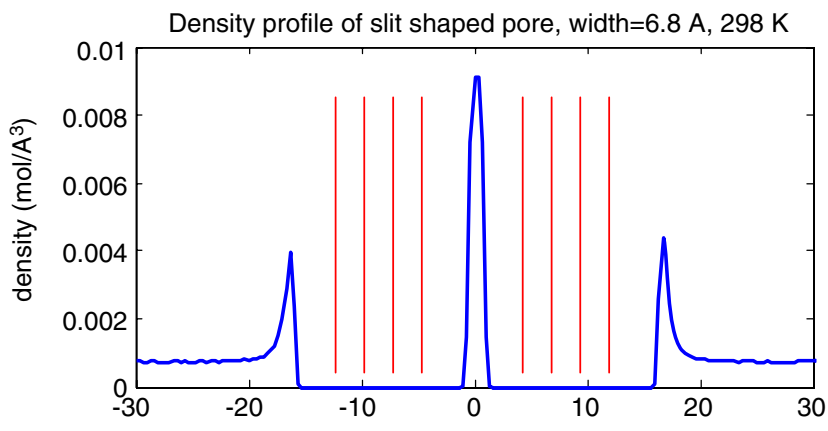

Density profile of slit shaped pore, width $=13.5 \mathrm{~A}, 298 \mathrm{~K}$

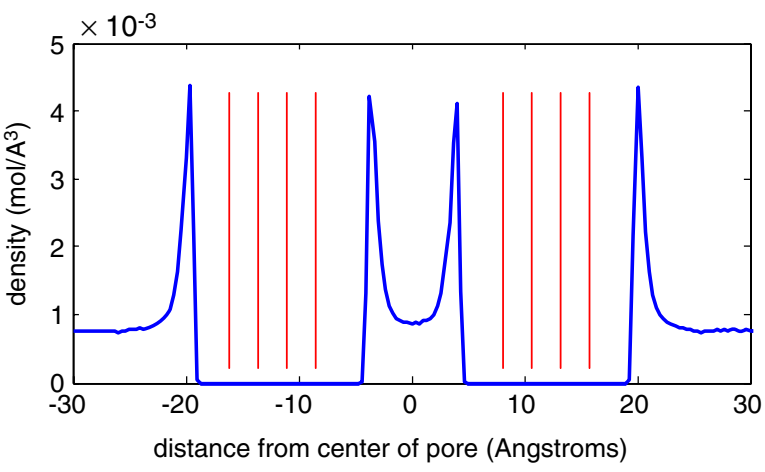

Figure 4. Laterally-averaged density profile for slit-shaped pores at 298 K. Narrower potentials have larger binding energies (and higher adsorption densities) but cannot host multiple $\mathrm{H}_{2}$ layers. The red vertical lines represent the positions of the graphene layers (see figure 3).

a range of pore widths and binding energies (sections 5 and 7); nevertheless it is worth noticing that narrower (higher $E_{\mathrm{B}}$ ) dominate the low-temperature and low-pressure adsorption, whereas larger pores (smaller $E_{\mathrm{B}}$ ) become more important at higher pressures and temperatures, as expected from basic statistical mechanics of adsorption.

Several models for adsorption of a gas on a solid at temperatures above the critical temperature, $T_{\mathrm{c}}$, above which no liquid phase exists at any pressure (for $\mathrm{H}_{2}, T_{\mathrm{c}}=33 \mathrm{~K}$ ) were investigated to predict $\mathrm{H}_{2}$ storage capacities in carbon nanopores. The model used is the Langmuir isotherm, in which 

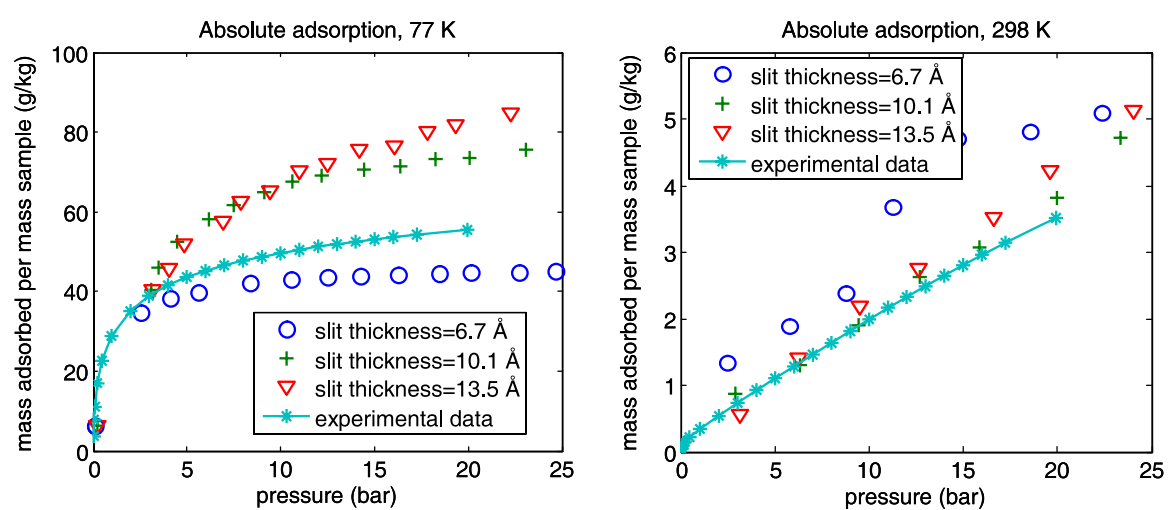

Figure 5. Absolute adsorption of $\mathrm{H}_{2}$ on nanoporous carbon: comparison of experimental and computational results (for various assumed pore sizes) at $77 \mathrm{~K}$ (left) and $298 \mathrm{~K}$ (right).

Table 2. Values of $\alpha(T)$ from computer simulations, and experimental upper bounds (equation (9)).

\begin{tabular}{rrrc}
\hline$T$ & $\alpha(T)^{\mathrm{a}}$ & $\frac{\theta(p, T) m \Sigma \cdot m_{\mathrm{s}} \mathrm{b}, \mathrm{c}}{m_{\mathrm{ads}}^{\mathrm{e}}(p, T)}$ & $\frac{m \Sigma \cdot m_{\mathrm{s}}}{m_{\mathrm{ads}}^{\mathrm{e}}(p, T)}$ \\
\hline $77 \mathrm{~K}$ & $6.4 \AA^{2}$ & $7.5 \AA^{2}(p=47$ bar, localized adsorption $)$ & $13.4 \AA^{2}(p=47 \mathrm{bar})$ \\
$293 \mathrm{~K}$ & $11.2 \AA^{2}$ & $15.1 \AA^{2}(p=47$ bar, mobile adsorption $)$ & $148 \AA^{2}(p=47 \mathrm{bar})$ \\
\hline
\end{tabular}

${ }^{a}$ Values from computer simulations.

${ }^{\mathrm{b}}$ Values calculated from experimental data for S-33/k in figure 1, extrapolated to $47 \mathrm{bar}$.

c $\theta(p, T)$ calculated from equations (6)-(8) with input for theoretical isotherms in figure 5 .

surface coverage varies with gas pressure $p$ and temperature $T$ according to [14]:

$$
\theta(p, T)=\frac{\chi(T) p}{1+\chi(T) p},
$$

where $\chi(T)$ is the Langmuir constant.

Two distinct models-localized adsorption and mobile adsorption-were used to estimate $\chi(T)$. In localized adsorption, the adsorbed $\mathrm{H}_{2}$ molecule has three vibrational degrees of freedom and oscillates around a minimum of the potential $V(x, y, z)$ (adsorption potential) where $z$ is the coordinate perpendicular to the surface, and $x, y$ are the coordinates parallel to the surface. The vibrational frequencies in the three directions, $v_{x}, v_{y}, v_{z}$, are usually of the order of $10^{12} \mathrm{~s}^{-1}$, and as a rule $v_{x} \approx v_{y}<v_{z}$. In mobile adsorption, the molecules still vibrate in the $z$ direction, but have free translational motion in the $(x, y)$ plane. The expressions for the Langmuir constant $\chi(T)$ for localized and mobile adsorption $\operatorname{are}^{6}$

$$
\begin{aligned}
\chi(T) & =\frac{\mathrm{e}^{E_{\mathrm{B}} /\left(N_{\mathrm{A}} k T\right)}}{\sinh \left(h v_{x} /(2 k T)\right) \sinh \left(h v_{y} /(2 k T)\right) \sinh \left(h v_{z} /(2 k T)\right)} \\
\times \sqrt{\frac{h^{6}}{(8 \pi m)^{3}(k T)^{5}}} & \\
\chi(T) & =\frac{\alpha(T) \mathrm{e}^{E_{\mathrm{B}} /\left(N_{\mathrm{A}} k T\right)}}{\sinh \left(h v_{z} /(2 k T)\right)} \sqrt{\frac{h^{2}}{8 \pi m(k T)^{3}}}
\end{aligned}
$$

6 For localized adsorption (equation (7)), harmonic oscillator type motion is assumed in all three directions. For mobile adsorption (equation (8)), the particles are free to move along the $(x, y)$ plane, but are bound by a harmonic oscillator potential along $z$ (perpendicular to the adsorption plane). respectively. In these expressions, $E_{\mathrm{B}}>0$ is the binding energy, defined as the depth of the minima of the potential energy $V(x, y, z)$ per mole of $\mathrm{H}_{2} ; m$ is the mass of the $\mathrm{H}_{2}$ molecule; $N_{\mathrm{A}}$ is Avogadro's constant; $k$ is Boltzmann's constant; and $h$ is Planck's constant. The surface area per site, $\alpha(T)$, is temperature-dependent because adsorbed molecules can be densely packed at low temperature, but occupy a larger area at higher temperature due to thermal motion in the $(x, y)$ plane. Note the informative inequality $\alpha(T)<$ (surface packing density) $^{-1}$ :

$$
\alpha(T)<\frac{\theta(p, T) m \Sigma \cdot m_{\mathrm{s}}}{m_{\mathrm{ads}}^{\mathrm{e}}(p, T)}<\frac{m \Sigma \cdot m_{\mathrm{s}}}{m_{\mathrm{ads}}^{\mathrm{e}}(p, T)} .
$$

The Langmuir constant $\chi(T)$ is large if the binding energy is large, the surface area per site is large, the vibrational frequencies are small, and/or the temperature is low. To calculate $\chi(T)$ for various scenarios of interest, and to investigate the sensitivity of the results to different estimates of $E_{\mathrm{B}}, v_{x}, v_{y}, v_{z}$, and $\alpha(T)$, the values collected in table 2 were used. The table shows that the values come from judicious combination of experimental and computational sources. The models were experimentally validated on sample S-33/k. Figure 6 shows excess adsorption isotherms calculated at 77 and $293 \mathrm{~K}$. The localized adsorption model at $77 \mathrm{~K}$ and the mobile adsorption model at $293 \mathrm{~K}$, calculated with $E_{\mathrm{B}}=5.3 \mathrm{~kJ} \mathrm{~mol}^{-1}$ (wide pores), agree qualitatively well with experimental data (figure 1) over a broad range of pressures. However, the Langmuir fit for $77 \mathrm{~K}$ falls below the experimental data at low pressures, consistent with the picture that sites with binding energies larger than 


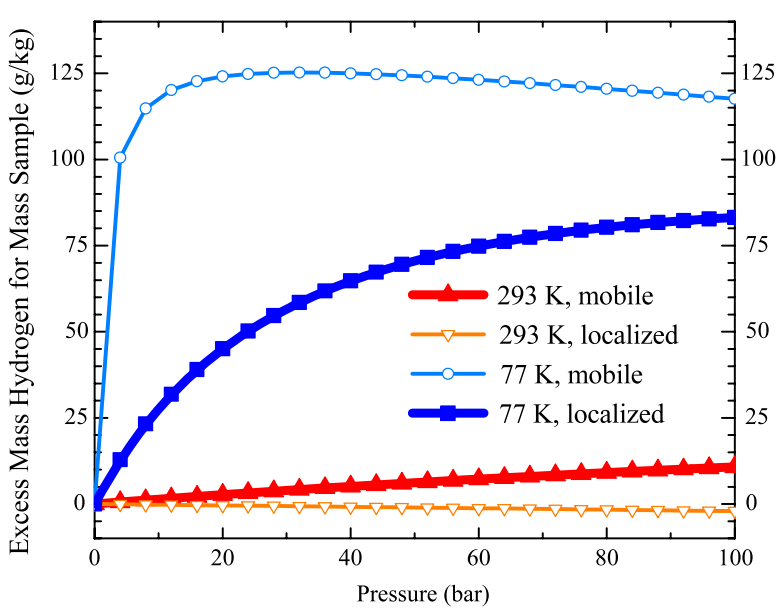

Figure 6. Theoretical $\mathrm{H}_{2}$ excess adsorption isotherms computed from the Langmuir isotherm for localized and mobile adsorption in sample S-33/k (equations (6)-(8)). Localized and mobile adsorption at $77 \mathrm{~K}$ and $293 \mathrm{~K}$, respectively (thick lines), are in good agreement with experimental data (figure 1). Localized and mobile adsorption at $293 \mathrm{~K}$ and $77 \mathrm{~K}$, respectively (thin lines), are ruled out by the experimental data. Excess adsorption drops with increasing pressure if the gas density increases more rapidly than the density of the adsorbed film, and turns negative (localized adsorption at $293 \mathrm{~K}$ ) if the gas density exceeds the film density.

$5.0 \mathrm{~kJ} \mathrm{~mol}^{-1}$, undoubtedly present in S-33/k, are occupied first and lead to higher coverage than what $5.0 \mathrm{~kJ} \mathrm{~mol}^{-1}$ entails (see also section 5). This agreement is remarkable in view of the experimental and theoretical input from widely different sources. Moreover, it allows the experimental discrimination between two vastly opposed situations of how molecules do or do not move along the surface, how such lateral dynamics (competition of vibrational and translational degrees of freedom) affects the $\mathrm{H}_{2}$ storage capacity, and how control of such dynamics, e.g., by surface functionalization, may offer new venues to increase storage capacities by as much as a factor of two.

\section{Hydrogen binding energy}

Figure 7 shows experimental absolute isotherms for samples S$33 / \mathrm{k}$ (left) and Batch 5.1 (right). The top row shows the best fit by a single Langmuir adsorption isotherm (equation (6), with a single binding energy). The bottom row shows a two binding energy fit:

$$
\begin{aligned}
& \frac{m_{\text {ads }}(p, T)}{m_{\mathrm{s}}}=\frac{m_{\text {ads }, p=\infty}}{m_{\mathrm{s}}}\left[\left(1-f_{\text {high }}\right) \theta\left(p, E_{\mathrm{B}, \text { low }}\right)\right. \\
& \left.\quad+f_{\text {high }} \theta\left(p, E_{\mathrm{B}, \text { high }}\right)\right],
\end{aligned}
$$

where $\theta$ is coverage, from which the low and high binding energy, $E_{\mathrm{B} \text {, low }}$ and $E_{\mathrm{B} \text {,high }}$, and fraction of high-energy sites, $f_{\text {high }}$. The binding energies from the fits are collected in table 3 . The room-temperature isotherms are so close to linear that they allow only for a low-pressure, high binding energy fit. The distinct values for $E_{\mathrm{B} \text {,high }}$ and $E_{\mathrm{B} \text {,low }}$ from the isotherms at cryogenic temperatures are remarkably similar in the two samples, considering how different the isotherms of the two samples look. The steep rise of Batch 5.1 at low pressure might suggest that $E_{\mathrm{B} \text {,high }}$ for Batch 5.1 is considerably larger than for S-33/k. However, table 3 shows that this is not so; instead the two samples have very similar high binding energies and a similar number of high energy binding sites per mass of sample. The values for $E_{\mathrm{B} \text {,high }}$ in table 3 from the cryogenic isotherms agree very well with the prediction obtained in section 4 . The values for $E_{\mathrm{B} \text {, low }}$ are somewhat on the high side compared to the prediction, which may be explained in terms of variations expected in the presence of surface defects and impurity atoms, which our samples may carry.

\section{Adsorption kinetics}

The kinetics was determined from the rate of adsorption onto the surface (also referred to as rate of condensation) and rate of desorption from the surface (also referred to as rate of evaporation), respectively, both in terms of surface coverage $\theta$. The rate of adsorption is given by

$$
\begin{aligned}
& \frac{\mathrm{d} \theta_{\mathrm{ads}}}{\mathrm{d} t}=\frac{\text { number of molecules adsorbed }}{(\text { number of surface sites }) \times \text { time }} \\
& \quad=\frac{p}{\sqrt{2 \pi m k T}}\left(1-\theta_{\mathrm{ads}}\right) \alpha(T),
\end{aligned}
$$

where $p /(2 \pi m k T)^{1 / 2}$ is the number of molecules striking the surface per unit area and time, at pressure $p$ and temperature $T$ (kinetic theory of gases; collisionless regime, in which the mean free path is large compared to the size of a pore); $1-\theta_{\text {ads }}$ is the fraction of unoccupied surface sites during adsorption (molecules adsorb only at sites that are not already occupied), and $\alpha(T)$ is the surface area per adsorption site as before. The rate of desorption is given by

$$
\frac{\mathrm{d} \theta_{\mathrm{des}}}{\mathrm{d} t}=\frac{\text { number of molecules desorbed }}{\text { (number of surface sites) } \times \text { time }}=\frac{\theta_{\mathrm{des}}}{\tau},
$$

where $\theta_{\text {des }}$ is the fraction of occupied surface sites during desorption (molecules desorb only from occupied sites), and $\tau$ is the adsorption lifetime [14]. The adsorption lifetime is obtained as follows. At equilibrium, the adsorption and desorption rate are equal, and so are the fractions of occupied sites at adsorption and desorption, $\theta_{\text {ads }}=\theta_{\text {des }}=\theta$, where $\theta$ is the equilibrium coverage. Solving the resulting equation for $\theta$ from (11) and (12), one finds:

$$
\theta=\frac{p \alpha(T) \tau / \sqrt{2 \pi m k T}}{1+p \alpha(T) \tau / \sqrt{2 \pi m k T}} .
$$

This is of the form of the Langmuir isotherm, equation (6). Equating the two formulae for $\theta$, equations (6), (13), and solving for $\tau$ gives the expression $\tau=(2 \pi m k T)^{1 / 2} \chi(T) / \alpha(T)$ for the lifetime, with $\chi(T)$ given by equations (7), (8), for localized and mobile adsorption, respectively. This makes equations (11), (12) first-order kinetic equations with completely specified coefficients. Their solutions as a function time of $t$, with initial conditions $\theta_{\text {ads }}(0)=0$ and $\theta_{\text {des }}(0)=1$, are

$\theta_{\text {ads }}(t)=1-\mathrm{e}^{-t / t_{\mathrm{ads}}}$,

$\theta_{\text {des }}(t)=\mathrm{e}^{-t / t_{\text {des }}}$ 

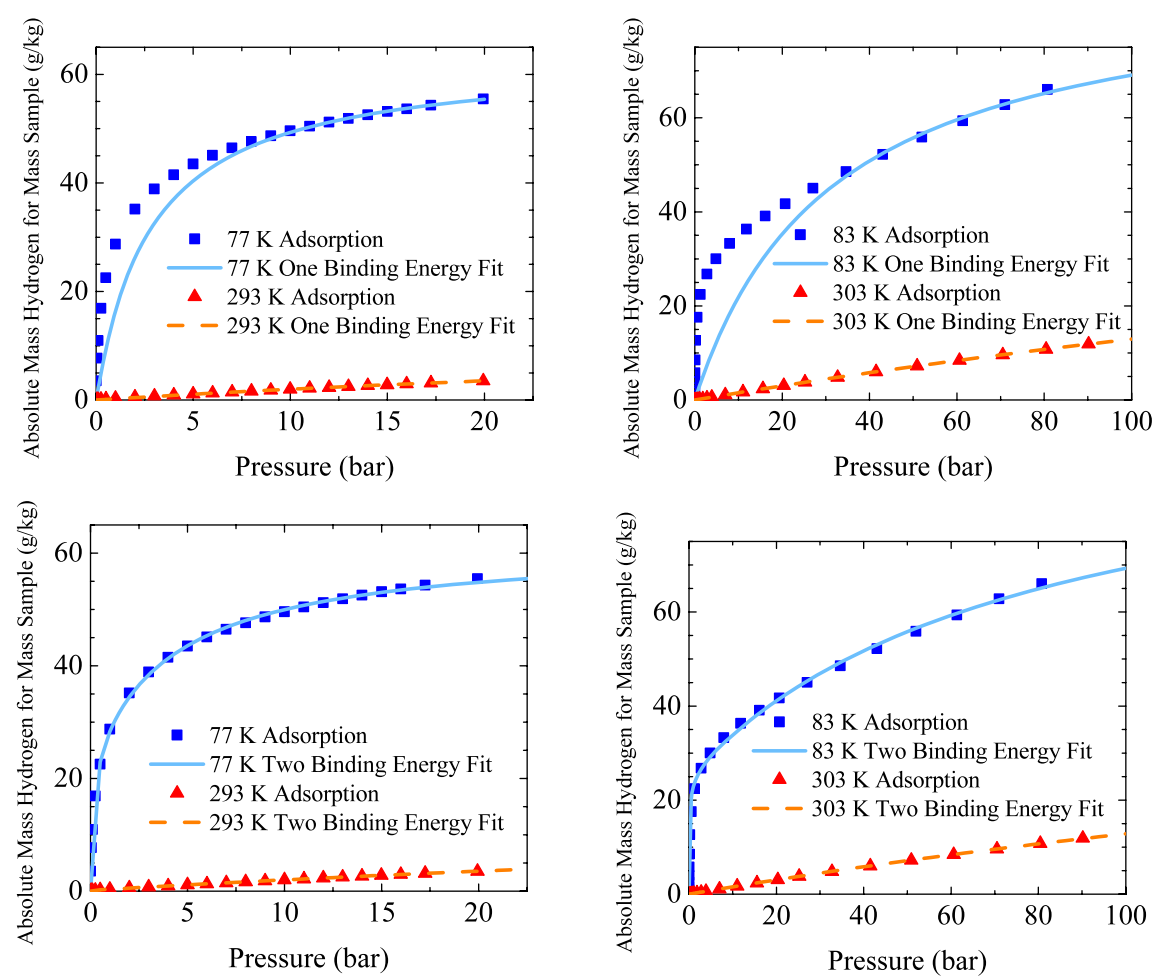

Figure 7. Absolute hydrogen adsorption for sample S-33/k (left) and Batch 5.1 (right). The BET surface areas were $2600 \mathrm{~m}^{2} \mathrm{~g}^{-1}$ and $3100 \mathrm{~m}^{2} \mathrm{~g}^{-1}$, respectively (table 5); the nanopore volumes (pores of width $\leqslant 10 \AA$ ) were 0.39 and $0.76 \mathrm{~cm}^{3} \mathrm{~g}^{-1}$ (table 5). Points are experimental; lines represent single-energy fits (top) and two-binding-energy fits (bottom, (equation (10)).

Table 3. Low and high binding energies and respective fractions of sites for samples S-33/k and Batch 5.1. The fraction of high-energy sites, $\sim 25-40 \%$, is consistent with a large fraction of the pore space residing in pores of width $\leqslant 10 \AA$. See also section 4 . Estimated uncertainties of binding energies and fraction of low/high-energy sites are less than $0.5 \mathrm{~kJ} \mathrm{~mol}^{-1}$ and $10 \%$, respectively.

\begin{tabular}{lllll}
\hline & $\begin{array}{l}\text { Low binding } \\
\text { energy }\end{array}$ & $\begin{array}{l}\text { Fraction of } \\
\text { low-energy sites }\end{array}$ & $\begin{array}{l}\text { High binding } \\
\text { energy }\end{array}$ & $\begin{array}{l}\text { Fraction of } \\
\text { high-energy sites }\end{array}$ \\
\hline S-33/k $(77 \mathrm{~K})$ & $6.4 \mathrm{~kJ} \mathrm{~mol}^{-1}$ & $61 \%$ & $8.6 \mathrm{~kJ} \mathrm{~mol}^{-1}$ & $39 \%$ \\
Batch $5.1(83 \mathrm{~K})$ & $4.8 \mathrm{~kJ} \mathrm{~mol}^{-1}$ & $75 \%$ & $9.0 \mathrm{~kJ} \mathrm{~mol}^{-1}$ & $25 \%$ \\
\hline
\end{tabular}

$$
\begin{aligned}
t_{\mathrm{ads}} & =\frac{\sqrt{2 \pi m k T}}{p \alpha(T)} \\
t_{\mathrm{des}} & =\sqrt{2 \pi m k T} \frac{\chi(T)}{\alpha(T)}
\end{aligned}
$$

(localized and mobile adsorption; all $T$ ),

$$
= \begin{cases}\frac{k T}{2 \pi m \alpha(T) v_{x} v_{y} v_{z}} \mathrm{e}^{E_{\mathrm{B}} /\left(N_{\mathrm{A}} k T\right)} & \text { localized; high } T \\ \frac{1}{v_{z}} \mathrm{e}^{E_{\mathrm{B}} /\left(N_{\mathrm{A}} k T\right)} & \text { mobile; high } T,\end{cases}
$$

where the coefficients in equations (11), (12) have been written as characteristic time scales for adsorption, $t_{\mathrm{ads}}$, and desorption, $t_{\text {des }}(=\tau)$, respectively. Figure 8 shows the surface coverage during adsorption and desorption as a function of time in units of the adsorption time $t_{\mathrm{ads}}$ and desorption time $t_{\mathrm{des}}$, respectively, and table 4 reports the values of adsorption and desorption time that were calculated from equations (16), (17) for cases of interest. The adsorption time is independent of the binding energy and whether adsorption is localized or mobile. It depends only on temperature and gas pressure, equation (16). The desorption time is independent of pressure. It depends on temperature, the binding energy, and whether adsorption is localized or mobile, equation (17). The desorption time increases with increasing strength of adsorption, $\chi(T)$, because $\mathrm{H}_{2}$ molecules are held more tightly to the surface, be it by increased binding energy (left to right in table 4), increased localization, or lower temperature (bottom to top in table 4).

The results in table 4 show that adsorption and desorption occur on very short time scales, between $\sim 10^{-11}$ s (adsorption at all $T$ ) and $\sim 10^{-7} \mathrm{~s}$ (desorption from mobile monolayer at $T=293 \mathrm{~K}$ and $E_{\mathrm{B}}=30 \mathrm{~kJ} \mathrm{~mol}^{-1}$ ), under all $\mathrm{H}_{2}$ storage conditions of interest. The short times can be understood as follows. For adsorption, the gas density at $p=50$ bar is high enough that the number of molecules striking the surface in one second, $p /(2 \pi m k T)^{1 / 2} \times \Sigma \times m_{\mathrm{s}} \times(1 \mathrm{~s})$, is approximately $10^{11}$ times larger than the number of molecules forming a monolayer on the surface, $\Sigma \times m_{\mathrm{s}} / \alpha(T)$. This gives $t_{\text {ads }} \sim 10^{-11} \mathrm{~s}$. For desorption from a mobile monolayer at ambient temperature, equation (18), the desorption time equals the period of oscillation of a molecule normal to the surface, divided by the probability that the molecule has energy $E_{\mathrm{B}}$ to leave the potential well, $\exp \left(-E_{\mathrm{B}} /\left(N_{\mathrm{A}} k T\right)\right)$. This gives 

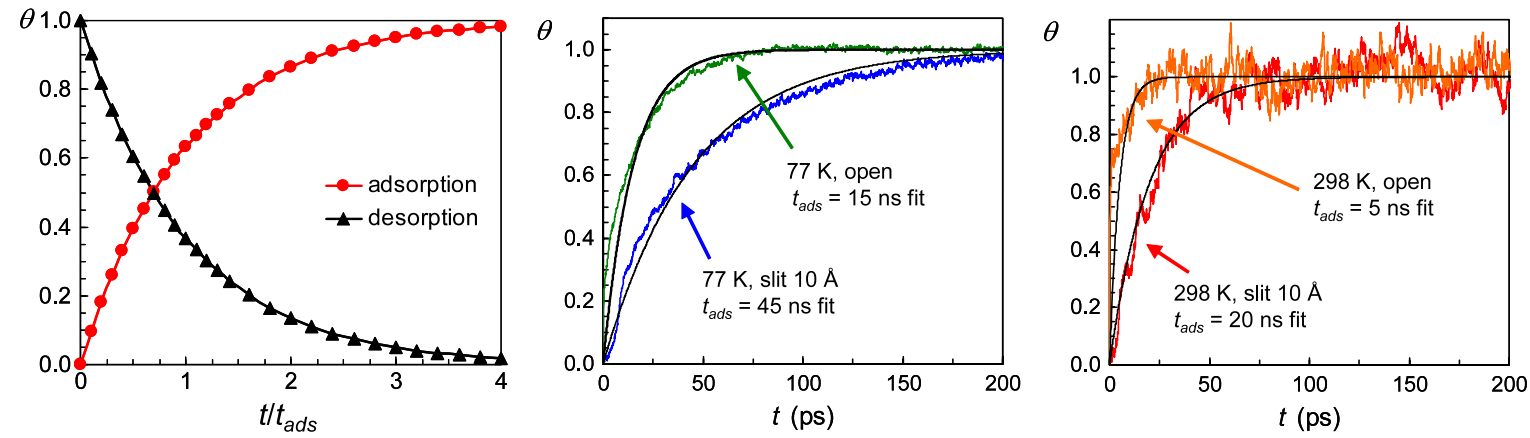

Figure 8. Left: time evolution of the surface coverage during adsorption and desorption (equations (14) and (15)). During adsorption, the surface is initially bare, exposed to $\mathrm{H}_{2}$ gas at pressure $p$ (equation (11)), and 95\% covered at time $t=4 t_{\text {ads }}$. During desorption, the surface is initially $100 \%$ covered, exposed to vacuum (equation (12)), and $95 \%$ bare at time $t=4 t_{\text {des }}$. Center and right: coverage during adsorption from the MD simulations (section 4) for open surfaces and slit-shaped pores at $77 \mathrm{~K}$ (center) and $298 \mathrm{~K}$ (right), and superimposed theoretical fits (equation (14)).

Table 4. Adsorption times, $t_{\mathrm{ads}}$, and desorption times, $t_{\mathrm{des}}$, from equation (16), (17), for localized and mobile adsorption. The pressure was set at $p=50$ bar throughout. The adsorption time increases or decreases at most by a factor of two if the pressure is lowered to 25 bar or raised to $100 \mathrm{bar}$, respectively. Desorption times for localized adsorption at $77 \mathrm{~K}$ and mobile adsorption at $293 \mathrm{~K}$, as observed in figures 1 and 6 , are highlighted in bold. The increase in desorption time with increasing temperature is small when the binding energy is small $\left(5.0 \mathrm{~kJ}\right.$ mol $\left.{ }^{-1}\right)$ and large when the binding energy is large $\left(30 \mathrm{~kJ} \mathrm{~mol}^{-1}\right)$. The ratio $t_{\text {des,localized }} / t_{\text {des,mobile }}$ is only weakly temperature-dependent and independent of the binding energy, equal to 0.058 and 0.13 at $77 \mathrm{~K}$ and $293 \mathrm{~K}$, and well approximated by the ratio $k T /\left(2 \pi m v_{x} v_{y} \alpha(T)\right)$ from equation (18).

\begin{tabular}{llll}
\hline & $E_{\mathrm{B}}=5.0 \mathrm{~kJ} \mathrm{~mol}^{-1}$ & $E_{\mathrm{B}}=14 \mathrm{~kJ} \mathrm{~mol}^{-1}$ & $E_{\mathrm{B}}=30 \mathrm{~kJ} \mathrm{~mol}^{-1}$ \\
\hline$T=77 \mathrm{~K}$ & & & \\
$t_{\text {ads }}$ & $1.5 \times 10^{-11} \mathrm{~s}$ & $1.5 \times 10^{-11} \mathrm{~s}$ & $1.5 \times 10^{-11} \mathrm{~s}$ \\
$t_{\text {des }}$, localized & $\mathbf{2 . 0} \times \mathbf{1 0}^{-11} \mathrm{~s}$ & $\mathbf{2 . 8} \times \mathbf{1 0}^{-\mathbf{5}} \mathrm{s}$ & $\mathbf{2 . 1} \times \mathbf{1 0}^{\mathbf{6}} \mathrm{s}$ \\
$t_{\text {des }}$, mobile & $5.3 \times 10^{-10} \mathrm{~s}$ & $7.3 \times 10^{-4} \mathrm{~s}$ & $5.6 \times 10^{7} \mathrm{~s}$ \\
$T=293 \mathrm{~K}$ & & & \\
$t_{\text {ads }}$ & $1.6 \times 10^{-11} \mathrm{~s}$ & $1.6 \times 10^{-11} \mathrm{~s}$ & $1.6 \times 10^{-11} \mathrm{~s}$ \\
$t_{\text {des }}$, localized & $2.5 \times 10^{-13} \mathrm{~s}$ & $1.0 \times 10^{-11} \mathrm{~s}$ & $7.6 \times 10^{-9} \mathrm{~s}$ \\
$t_{\text {des }}$, mobile & $\mathbf{2 . 1} \times \mathbf{1 0}^{-\mathbf{1 2}} \mathrm{s}$ & $\mathbf{8 . 5} \times \mathbf{1 0}^{-\mathbf{1 1}} \mathrm{s}$ & $\mathbf{6 . 2} \times \mathbf{1 0}^{-\mathbf{8}} \mathrm{s}$ \\
\hline
\end{tabular}

$t_{\text {des }} \sim 10^{-12} \mathrm{~s}$, for $E_{\mathrm{B}}=5.0 \mathrm{~kJ} \mathrm{~mol}^{-1}$ and $T=293 \mathrm{~K}$, because the oscillation frequency $v_{z}$ is of the order $10^{12} \mathrm{~Hz}$.

The short adsorption/desorption characteristic times imply that this process will not determine how fast $\mathrm{H}_{2}$ can be loaded/unloaded into/from the nanoporous carbon even if surface functionalization increased the binding energies in a significant way. The general shape and characteristic times for adsorption are in good agreement with the MD simulations (figure 8, right).

\section{Sample characterization}

Nitrogen adsorption experimental isotherms from $10^{-7}$ to 1 bar and $77 \mathrm{~K}$ (sub-critical temperature and pressure) were run on the activated carbons using a Quantachrome Instruments Autosorb-1-C. Nitrogen adsorption allows the characterization of pores in the micropore regime and mesopore regime [15]. Two methods of analysis were performed on the nitrogen isotherms: Brunauer, Emmett, Teller (BET) theory of adsorption [16] and non-local density functional theory (NLDFT) [17]. A list of our typical BET surface areas are given in table 5 .

As a reference for material properties, BET surface area is useful, but has been shown to be limited for predominantly
Table 5. Specific surface areas of samples. BET surface areas are found from the pressure range of $0.01-0.03 p / p_{0}$, DFT is for slit-widths $<36 \mathrm{~nm}$. For BET, a cross sectional area of $0.162 \mathrm{~nm}^{2}$ is used for $\mathrm{N}_{2}$ [18].

\begin{tabular}{lll}
\hline & \multicolumn{2}{c}{ Sample } \\
\cline { 2 - 3 } & Batch 5.1 & $\mathrm{S}-33 / \mathrm{k}$ \\
\hline BET surface area $\left(\mathrm{m}^{2} \mathrm{~g}^{-1}\right)$ & 3100 & 2600 \\
NLDFT & & \\
Surface area $\left(\mathrm{m}^{2} \mathrm{~g}^{-1}\right)$ & 3200 & 2200 \\
Total pore volume $\left(\mathrm{cm}^{3} \mathrm{~g}^{-1}\right)$ & 1.69 & 1.09 \\
Porosity $(\%)$ & 77 & 69 \\
Pore volume $<10 \AA\left(\mathrm{cm}^{3} \mathrm{~g}^{-1}\right)$ & 0.76 & 0.39 \\
Pore width mode $(\AA)$ & 7.5 & 11.3 \\
\hline
\end{tabular}

microporous samples [19]. Non-local density functional theory presents an opportunity to better model gas behavior in micropores $[15,20]$. This microscopic approach is used to determine isotherms for individual pore sizes and shapes, which are then compared to the experimental isotherm using the integral equation

$$
N\left(p / p_{0}\right)=\int_{D_{\mathrm{MIN}}}^{D_{\mathrm{MAX}}} N\left(p / p_{0}, D\right) f(D) \mathrm{d} D .
$$




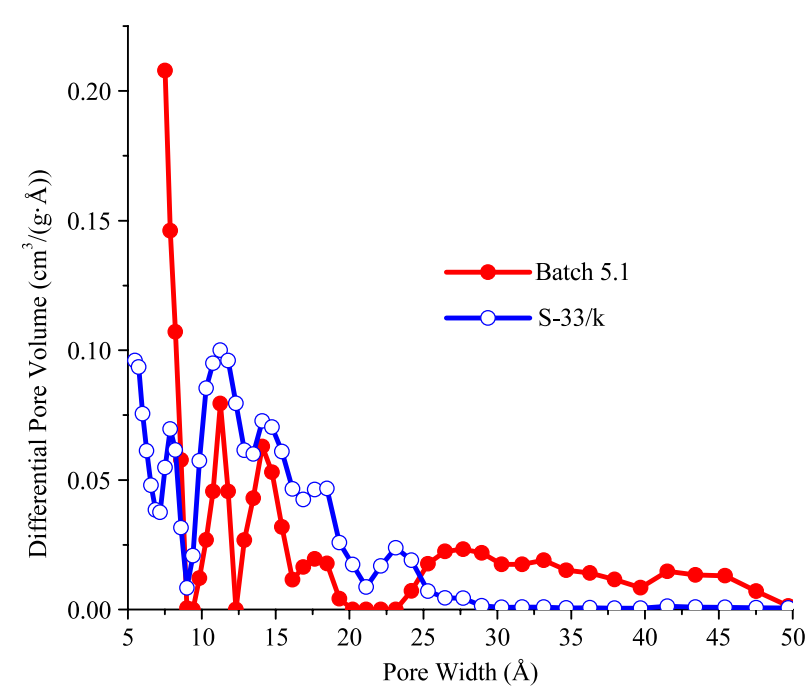

Figure 9. Pore size distribution calculated using NLDFT of samples Batch 5.1 and S-33/k. Periodicity and agreement of peaks suggests a physical cause, for example broken graphene sheets acting as splints between the graphene layers. Batch 5.1 exhibits a larger volume of nanopores and surface area (table 5), but does not have as high of a hydrogen uptake as $\mathrm{S}-33 / \mathrm{k}$. This may be due to the influence of pores larger than $1 \mathrm{~nm}$, i.e. larger pores than $1 \mathrm{~nm}$ may be required for two layers of hydrogen to adsorb.

In this expression, $D$ is the pore dimension (width of slit), $N\left(p / p_{0}\right)$ is the experimental isotherm, $N\left(p / p_{0}, D\right)$ is the isotherm of a single pore dimension found by NLDFT and $f(D)$ is the relative distribution of these widths. This model was applied to the nitrogen adsorption isotherms using the Quantachrome software for slit-shaped pores. This provides pore size distributions of pore volume and surface area for pores of less than $36 \mathrm{~nm}$. This upper limit to the pore size using NLDFT is still a better judge of the actual surface area, since it is dominated by the area due to micropores. Figure 9 and table 5 lists pore surface areas, pore volumes and porosity from NLDFT. The pore sizes and distribution are consistent with small-angle x-ray scattering (SAXS) analysis of our samples conducted at the Advanced Photon Source, Argonne National Laboratory [9].

\section{Conclusions}

This paper demonstrates that high-surface-area activated carbon materials based on ground corncob (an agricultural waste byproduct), offer promise for reversible storage of hydrogen for vehicular use. Storage capacities of up to $100 \mathrm{~g}$ $\mathrm{H}_{2} / \mathrm{kg}\left(90\right.$ bar, $83 \mathrm{~K}$ ) and $20 \mathrm{~g} \mathrm{H}_{2} / \mathrm{kg}$ (90 bar, $303 \mathrm{~K}$ ) were demonstrated experimentally. The experiments hereby presented, combined with computational simulations and thermodynamics theory are able to discriminate between two different conditions of adsorption (mobile versus localized); the engineering of such conditions could lead to doubling of storage capacities in future materials. The paper also presents the characterization of the experimental adsorption isotherms in terms of low and high binding energies, consistent with the $\mathrm{H}_{2}$-substrate interaction for subnanometer sized pores.
Finally, the agreement between simple activation kinetics and molecular dynamics simulations of adsorption in nanopores show that the process is very fast and highly reversible, highlighting the fact that the kinetics of the $\mathrm{H}_{2}$ tank will be limited by other physical processes.

\section{Acknowledgments}

This material is based upon work supported in part by the Department of Energy under Award No. DE-FG0207ER46411. Use of the Advanced Photon Source was supported by the US Department of Energy, Office of Science, Office of Basic Energy Sciences, under Contract No. DEAC02-06CH11357. CW and RC gratefully acknowledge the University of Missouri Bioinformatics Consortium for the use of their computational facilities. The authors would like to thank M Frederick Hawthorne, Francisco RodríguezReinoso, Louis Schlapbach, Andreas Züttel, Bogdan Kuchta, Lucyna Firlej, Michael Roth, and Michael Gordon for valuable contributions. Finally, the authors would like to acknowledge helpful contributions by Hiden Isochema Ltd, Warrington, UK.

\section{References}

[1] Satyapal S, Petrovic J, Read C, Thomas G and Ordaz G 2007 Catal. Today $120246-56$

[2] See also http://www.sc.doe.gov/bes/hydrogen.pdf (May 2003), http://www1.eere.energy.gov/hydro-genandfuelcells/mypp/ (Feb. 2005), http://www.hydrogen.energy.gov/ annual_review06_proceedings.html (May 2006)

[3] Bhatia S K and Myers A L 2006 Langmuir 22 1688-700

[4] Patchovskii S, Tse J S, Yurchenko S N, Zhechkov L, Heine T and Seifert G 2005 Proc. Natl Acad. Sci. USA 102 10439-44

[5] Ferro Y, Marinelli F, Allouche A and Brosset C 2003 J. Chem. Phys. 118 5650-7

Zhao Y, Kim Y H, Dillon A C, Heben M J and Zhang S B 2005 Phys. Rev. Lett. 94155504

Kim Y H, Zhao Y, Williamson A, Heben M J and Zhang S B 2006 Phys. Rev. Lett. 96016102

Chung M, Crespi V, Eklund P and Foley H 2006 DOE Hydrogen Program Review ST27

[6] Pfeifer P, Suppes G J, Shah P S and Burress J W 2007 US Patent Application 11/937,150

[7] Poirier E, Chahine R, Bénard P, Cossement D, Lafi L, Mélançon E, Bose T K and Désilets S 2004 Appl. Phys. A 78961

Bénard P and Chahine R 2007 Scr. Mater. 56803

[8] Furukawa H, Miller M A and Yaghi O M 2007 J. Mater. Chem. 173197

[9] Pfeifer P et al 2008 Mater. Res. Soc. Symp. Proc. 1041 1041-R02-02

[10] Myers A L and Monson P A 2002 Langmuir 1810261

[11] Phillips J C, Braun R, Wang W, Gumbart J, Tajkhorshid E, Villa E, Chipot C, Skeel R D, Kale L and Schulten K 2005 J. Comput. Chem. 261781 See also http://www.ks.uiuc.edu/ Research/namd/

[12] Connolly M J, Roth M W, Gray P A and Wexler C 2008 Langmuir 243228

Firlej L, Kuchta B, Roth M W, Connolly M J and Wexler C 2008 Langmuir 2412392

[13] Mattera L, Rosatelli F, Salvo C, Tommasini F, Valbusa U and Vidali G 1980 Surf. Sci. 93515

[14] Bruch L W, Cole M W and Zaremba E 1997 Physical Adsorption: Forces and Phenomena (Oxford: Clarendon) 
[15] Dabrowski A 2003 Particle Particle Syst. Characterization 20311

[16] Brunauer S, Emmett P E and Teller E 1938 J. Am. Chem. Soc. 603309

[17] Lastoskie C, Gubbins K E and Quirke N 1993 J. Phys. Chem. 974786

Gubbins K E 1997 Physical Adsorption: Experiment, Theory and Application ed J Fraissard (Dordrecht: Kluwer) Dombrowski R J, Hyduke D R and Lastoskie C M 2000 Langmuir 165041
[18] Lowell S, Shields J, Charalambous G and Manzione J 1982 J. Colloid Interface Sci. 86191

[19] Rouquerol F, Rouquerol J and Sing K 1999 Adsorption by Powders and Porous Solids: Principles, Methodology and Applications (New York: Academic)

[20] Gelb L D, Gubbins K E, Radhakrishnan R and Sliwinska-Bartkowiak M 1999 Rep. Prog. Phys. 621573 Ravikovitch P I, Vishnyakov A, Russo R and Neimark A V 2000 Langmuir 162311 